\title{
(C) OPEN ACCESS \\ Targeted therapy for the post-operative conjunctiva: SPARC silencing reduces collagen deposition
}

\author{
Li Fong Seet, ${ }^{1,2,3}$ Yang Fei Tan, ${ }^{4}$ Li Zhen Toh, ${ }^{1}$ Stephanie WL Chu, ${ }^{1}$ Ying Shi Lee, ${ }^{1}$ \\ Subbu S Venkatraman, ${ }^{1,4,5,6}$ Tina T Wong ${ }^{1,2,3,4,7}$
}

${ }^{1}$ Ocular Therapeutics and Drug Delivery, Singapore Eye Research Institute, Singapore, Singapore

2Department of Ophthalmology, Yong Loo Lin School of Medicine, National University of Singapore, Singapore, Singapore ${ }^{3}$ Duke-NUS Medical School, Singapore, Singapore

${ }^{4}$ School of Materials Science and Engineering, Nanyang

Technological University, Singapore, Singapore

${ }^{5}$ NTU - Northwestern University Institute for Nanomedicine, Singapore, Singapore

${ }^{6}$ MedTech, National Heart Centre, Singapore, Singapore ${ }^{7}$ Glaucoma Service, Singapore National Eye Centre, Singapore, Singapore

\section{Correspondence to}

Professor Tina T Wong, Glaucoma Service, Singapore National Eye Centre, Singapore 168751, Singapore; tina. wong.t.|@singhealth.com.sg and Subbu S Venkatraman, School of Materials Science and Engineering, Nanyang Technological University, Singapore 639798, Singapore; ASSubbu@ntu.edu.sg

LFS and YFT contributed equally.

Received 17 January 2018 Revised 22 April 2018 Accepted 24 May 2018 Published Online First 18 July 2018

\section{ABSTRACT \\ Background To develop targeted antifibrotic therapy for glaucoma filtration surgery; this study determines the effectiveness of small interfering RNA (siRNA) to reduce in vivo secreted protein acidic and rich in cysteine (SPARC) expression using the mouse model of conjunctival scarring.}

Methods Experimental surgery was performed as described for the mouse model of conjunctival scarring. Scrambled (siScram) or Sparc (siSparc) siRNAs, loaded on layer-by-layer (LbL) nanoparticles, were injected into the conjunctiva immediately after surgery. Expression of Sparc, Col1a1, Fn1 and Mmp14 was measured by real-time PCR and immunoblotting on days 7 and 14 postsurgery. Live imaging of the operated eyes was performed using slit lamp, anterior segment-optical coherence tomography and confocal microscopy. Tissue pathology was evaluated by histochemical and immunofluorescent analyses of operated conjunctival cryosections. Tissue apoptosis was quantitated by annexin $\mathrm{V}$ assay.

Results siSparc, delivered via expanded LbL nanoparticles, significantly inhibited Sparc transcription in both day 7 (2.04-fold) and day 14 (1.39-fold) treated tissues. Sparc suppression on day 7 was associated with a significant reduction of Col1a1 (2.52-fold), Fn1 (2.89fold) and Mmp 14 (2.23-fold) mRNAs. At the protein level, both SPARC and collagen 1A1 (COL1A1) were significantly reduced at both time points with siSparc treatment. Nanoparticles were visualised within cell-like structures by confocal microscopy, while overt tissue response or apoptosis was not observed.

Conclusions SPARC targeted therapy effectively reduced both SPARC and collagen production in the operated mouse conjunctiva. This proof-of-concept study suggests that targeted treatment of fibrosis in glaucoma surgery is safe and feasible, with the potential to extend to a range of potential genes associated with fibrosis.

\section{INTRODUCTION}

The major benefits of targeted therapy include reduced adverse effects on unaffected tissues and the promise of increased effectiveness in achieving the therapeutic goal. Precise knowledge of the molecular pathways being modulated also allows for accurate evaluation of therapeutic effectiveness and efficiency and facilitates improved therapeutic strategies with other synergistic/combinatorial treatments. Moreover, individualised treatment is possible based on the unique genetic predisposition of each patient.
In glaucoma filtration surgery (GFS), subconjunctival fibrosis over the sclerostomy site occurs to varying degrees and is a major cause of surgical failure. ${ }^{1}$ Antiscarring agents are routinely applied as surgical adjuncts to reduce the fibroproliferative response and improve the success rates of the surgery. For this purpose, mitomycin $\mathrm{C}$ (MMC) is the most commonly used adjunct. Although MMC is highly effective in reducing the postoperative scarring response, surgeries continue to fail. ${ }^{2}$ Moreover, its non-specific and irreversible properties increase the rate of sight-threatening complications including infection and hypotony. ${ }^{3}$ Furthermore, some patients appear to develop resistance to MMC treatment. ${ }^{4}$ Hence, fibrosis in GFS is a condition that may benefit greatly from targeted therapy, either alone or in conjunction with current antifibrotic therapy to overcome drug resistance and/or reduce adverse side effects.

In this study, we explore the feasibility of a targeted approach to antifibrotic therapy for the operated conjunctiva via small interfering RNA (siRNA). The target gene is the $32 \mathrm{kDa}$, secreted protein acidic and rich in cysteine (SPARC), also known as osteonectin and BM-40. SPARC is a prototypic calcium-binding matricellular protein. ${ }^{5}$ Matricellular proteins are secreted glycoproteins that are largely non-structural and involved in mediating cellular interactions with components of the extracellular matrix (ECM). ${ }^{6}$ SPARC is conspicuously induced at sites of wound healing and tissue remodelling, including the wounded conjunctiva. ${ }^{7}$ Conversely, when SPARC is absent, as in the SPARC knockout mouse, altered collagen fibrillogenesis and delayed fibrosis was observed. ${ }^{8}$ Indeed, we have also found that SPARC-/- mice displayed reduced fibrosis in the mouse model of conjunctival scarring. ${ }^{9}$ A likely mechanism for this antifibrotic response is the reduced deposition of collagen which was assembled from collagen fibrils that were thinner than normal. ${ }^{9}$ We have since confirmed that collagen expression and fibril thickness in the SPARC-null mouse is also significantly lower than normal in other parts of the eye, such as the iris. ${ }^{10}$ Although collagen is thought to be the key protein regulated by SPARC ${ }^{11}$ loss of SPARC was also associated with reduced deposition of other extracellular matrix components such as fibronectin (FN) and matrix metalloproteinases (MMP-2 and MMP-14) in the operated conjunctiva. ${ }^{9}$ All these data support the targeting of SPARC expression as a means to 
reduce deposition of collagen and other extracellular matrix proteins and is therefore a potential antifibrotic therapeutic strategy.

We have previously established that Sparc expression was amenable to silencing by small interfering RNA (siRNA) in primary mouse conjunctival fibroblasts and this inhibition was associated with reduced collagen expression. ${ }^{12}$ As a method for siRNA delivery, we have shown that layer-by-layer (LbL) nanoparticles comprising of poly-L-arginine (ARG) atop a hydroxyapatite (HA) core successfully silenced Sparc expression in conjunctival fibroblasts. ${ }^{13}$ In this study, we further demonstrate that treatment with Sparc siRNA-loaded LbL nanoparticles in the mouse model of conjunctival scarring effectively reduced production of SPARC and extracellular matrix proteins, particularly collagen. While SPARC and collagen suppression remained effective for at least 2 weeks post-treatment, the control of other extracellular proteins including FN and MMP-14 was effective mainly in the short term. Finally, we did not find apparent tissue toxicity or tissue response in the operated mouse conjunctiva treated with the siRNA-loaded nanoparticles. Overall, our data suggest that genetic inhibition by means of siRNA-based gene silencing is a promising targeted strategy for ameliorating conjunctival fibrosis after GFS.

\section{MATERIALS AND METHODS}

\section{Mouse model of conjunctival scarring}

All experiments with animals were treated in accordance with the Association for Research in Vision and Ophthalmology (ARVO) Statement on the Use of Animals in Ophthalmic and Vision Research. Wild type C57BL6/J mice, bred and maintained at the Department of Experimental Surgery (Singapore General Hospital, Singapore), were used. Experimental surgery, resulting in conjunctival scarring, was performed on the left eye of each mouse to induce scarring as described previously. ${ }^{9}$ Mice were anaesthetised by intraperitoneal injection of a 0.1 $\mathrm{mL}$ ketamine/xylazine mixture containing $2 \mathrm{mg} / \mathrm{mL}$ xalazine hydrochloride (Troy Laboratories, Smith-field, Australia) and $20 \mathrm{mg} / \mathrm{mL}$ ketamine hydrochloride (Ketamine, Parnell Laboratories, Alexandria, Australia) as well as topically with one drop of $1 \%$ xylocaine per eye before surgery was performed. The conjunctiva was surgically dissected to expose the underlying sclera, after which an incision was made through the sclera into the anterior chamber of the eye with a 30 -gauge needle to create a fistula. The wound was then secured and closed at the limbus by a $10-0(0.2$ metric) Ethilon black monofilament nylon scleral suture (Ethicon). LbL nanoparticles in $5 \mu \mathrm{L}$ phosphate-buffered saline (PBS), loaded with either scrambled or Sparc siRNA, were administered by direct injection into the conjunctiva at the surgical site immediately after surgery. Fucithalmic ointment (Leo Pharmaceutical Products, Ballerup, Denmark) was instilled at the end of the procedure to prevent infection. For molecular evaluation of tissues on days 7 or 14 after surgery, mice were euthanised by intraperitoneal injection of pentobarbitol sodium, at $100 \mathrm{mg} / \mathrm{kg}$ body weight, before conjunctival tissues were harvested.

\section{SiRNA}

The siRNAs used in this study were: scrambled control siRNA 5'-GCUCACAGCUCAAUCCUAAUC-3', Sparc siRNA 5'AACAAGACCUUCGACUCUUCC- ${ }^{\prime}$. These siRNAs were custom synthesised and purchased from Bioneer Corp. (South Korea).

\section{Preparation of siRNA LbL nanoparticles}

The LbL nanoparticle fabrication was performed as described previously. ${ }^{13} \mathrm{HA}$ nanoparticles at $10 \mathrm{mg} / \mathrm{mL}$ was added to ARG solution at $0.5 \mathrm{mg} / \mathrm{mL}$, in a volume ratio of $1: 10$, with maximum vortex mixing and light sonication for $10 \mathrm{~min}$. The ARG-coated nanoparticles were suspended in $0.1 \mathrm{M} \mathrm{NaCl}$ and mixed with 1.2 pmol of siRNA per $\mu \mathrm{g}$ HA for 1 hour. Another layer of ARG was added by mixing with the $0.5 \mathrm{mg} / \mathrm{mL}$ ARG solution. This method will generate the three-layer configuration (HA-ARG-siRNA-ARG) ${ }^{13}$ To obtain the five-layer configuration (HA-ARG-siRNA-ARG-siRNA-ARG), the above coating steps were repeated with 1.2 pmol of siRNA per $\mu \mathrm{g}$ HA and ending with a final layer of ARG. The nanoparticles were resuspended in sterile PBS and stored at $4^{\circ} \mathrm{C}$ before use. $\mathrm{LbL}$ nanoparticles loaded with siScram are labelled as siScram-LbL while those loaded with siSparc are labelled as siSparc-LbL in the text and figure legends.

\section{Real-time quantitative PCR (qPCR) analysis}

Operated conjunctival tissues injected with siRNA-loaded nanoparticles from five mice were pooled as one group in a total of five groups for each condition $(n=5)$. Tissues from the contralateral untreated eyes in each group of mice were similarly pooled and used as baseline for calculation of fold change in mRNA expression in each group. Conjunctival tissues were processed and analysed by qPCR as described previously. ${ }^{7}$ All qPCR reactions were performed in triplicate. All mRNA levels were measured as CT threshold levels. Rpl13a was the used as the housekeeping gene for days 7 and 14 operated conjunctival tissues injected with the siRNAloaded LbL nanoparticles. Values were calculated as fold changes relative to similarly pooled contralateral, unoperated conjunctival tissues by the $2-\Delta \Delta C T$ method. The primers used were as follows: Rpl13a-forward 5'-GAGGTCGGGTGGAAGTACCA-3' and Rpl13a-reverse 5'-TGCATCTTGGCCTTTTCCTT-3'; Sparc-forward 5'-AAACATGGCAAGGTGTGTCA-3' and Sparc-reverse 5'-AAGTGGCAGGAAGAGTCGAA-3'; Col1a1-forward 5'-CCCACCCCAGCCGCAAAGAG-3' and Col1a1-reverse $\quad 5^{\prime}$-GCCATGCGTCAGGAGGGCAG-3'; Fn1-forward $5^{\prime}$ - ATGTGGACCCCTCCTGATAGT-3' and Fn1-reverse 5'-GCCCAGTGATTTCAGCAAAGG-3'; Mmp14-forward $5^{\prime}$-TGGCATCCAGCAACTTTATG-3' and Mmp14-reverse 5'-TTTGGGCTTATCTGGGACAG-3'.

\section{Immunoblotting}

Operated conjunctival tissues from mice treated with siRNAloaded nanoparticles were harvested after 7 or 14 days and processed as described previously. ${ }^{7}$ Operated conjunctival tissues from five mice were pooled as one group in a total of three groups for each condition $(n=3)$. Antibodies against SPARC, collagen 1A1 (COL1A1), FN1, MMP-14 and glyceraldehyde 3-phosphate dehydrogenase (GAPDH) were purchased from Cell Signalling Technology (Massachusetts, USA), Abnova Corp. (Colorado, USA), Epitomics (Abcam, Massachusetts, USA), Abcam (Massachusetts, USA) and Santa Cruz Biotechnology (California, USA), respectively. Horseradish peroxidase-conjugated secondary antibodies were from Jackson ImmunoResearch Laboratories (Pennsylvania, USA). Densitometric quantitation was performed using Image Studio Lite V.5 (LI-COR Biosciences, Nevada, USA). Normalisation to correct for variations in loading was performed using GAPDH as the housekeeping protein. 


\section{In vivo imaging of mouse eyes}

Operated mice were anaesthetised by intraperitoneal injection of a $0.1 \mathrm{~mL}$ ketamine/xylazine mixture containing $2 \mathrm{mg} / \mathrm{mL}$ xalazine hydrochloride (Troy Laboratories, Smith-field, Australia) and 20 $\mathrm{mg} / \mathrm{mL}$ ketamine hydrochloride (Ketamine) before imaging was performed. Slit-lamp microscopy was performed using Righton LED slit lamp MW50D (Right Mfg Co Ltd, Japan). Anterior segment-optical coherence tomography (AS-OCT) was carried out using the Optovue RTVue100-2 Fourier domain optical coherence tomography system (Optovue, California, USA). In vivo confocal microscopic examinations of the operated and treated conjunctiva were performed using Hrt3 microscope (Heidelberg Engineering, Heidelberg, Germany).

\section{Histochemical and immunofluorescent evaluation of cryosections}

Histochemical evaluation of operated conjunctival cryosections by H\&E or picrosirius red staining was performed as described previously. ${ }^{7}$ Polarisation microscopy was performed using the Nikon Eclipse Ti microscope (Nikon Instruments, New York, USA). For immunofluorescent analyses, labelling by primary antibodies for SPARC (sc59703; Santa Cruz Biotechnology), COL1A1 (H00001277-M01; Abnova, Taiwan), FN1 (1574-1; Epitomics/Abcam) or MMP-2 (250752; Abbiotec, California, USA) was detected using secondary goat antibodies conjugated to Alexa Fluor-488 (A11001; green fluorescence) or Alexa Fluor-594 (A11012; red fluorescence), both obtained from Invitrogen Corp. (Thermo Fisher Scientific, Massachusetts, USA). Nuclei were visualised by mounting the cells in 4',6-diamidino-2-phenylindole-containing Vectashield mounting medium (Vector Laboratories, California, USA). Labelled cells were visualised using the Zeiss Imager.Z1 microscope (Carl Zeiss, USA).

\section{Annexin V assay by flow cytometry}

Apoptosis in the operated conjunctival tissues was measured using the Guava Nexin Reagent (Guava Technologies, Hayward, California, USA) by flow cytometry according to manufacturer's instructions. Cells were isolated for flow cytometry as described previously. ${ }^{7}$ Five thousand cells were analysed for each sample in a total of five samples for each condition $(n=5)$. Cell populations were quantified using the Guava Nexin software (EMD Millipore, Massachusetts, USA).

\section{Statistical analysis}

Data are expressed as mean \pm SD. For pairwise comparison of fold mRNA or protein expression between siScram-LbL and siSparc-LbL treated tissues, the significance of differences between the two conditions was determined by the two-tailed Student's t-test. For comparison of \%Annexin V+cells in tissues between different treatment conditions, the significance of differences between the time points or treatments was determined by one-way analysis of variance with Bonferroni post-hoc adjustment using SPSS statistics. A p value of $<0.05$ was deemed to be significant.

\section{RESULTS}

Downregulation of SPARC and extracellular matrix molecules in the operated conjunctiva treated with siSparc-loaded nanoparticles

We have previously shown that LbL nanoparticles containing one layer of Sparc siRNA (siSparc) was effective in silencing Sparc expression in cultured conjunctival fibroblasts. ${ }^{13}$ Hence, we first determined whether nanoparticles in this three-layered format was effective for delivering Sparc silencing in the mouse model of conjunctival scarring after 7 days of treatment. The three-layered LbL nanoparticles were $275 \pm 2 \mathrm{~nm}$ in hydrodynamic diameter with a surface charge of $47 \pm 5 \mathrm{mV}$ and polydispersity of $0.26 \pm 0.02$ (data not shown). These particles consisted of 1.2 pmol of siRNA per $\mu \mathrm{g} \mathrm{HA} / \mathrm{siRNA}$ layer, enabling the injection of 3208 ng siRNA per mouse eye. When compared with tissues injected with scrambled siRNA (siScram), tissues treated with siSparc demonstrated no significant alteration of either Sparc or Col1a1 mRNA expression (data not shown). These data suggest that the three-layered nanoparticles did not have the capacity to deliver specific Sparc silencing through their siRNA payload in the operated conjunctiva.

To investigate whether greater quantities of siRNA may be required to bring about Sparc silencing in vivo, we fabricated five-layered LbL nanoparticles containing an additional layer of siSparc (figure 1A). The five-layered LbL nanoparticles were $242 \pm 2 \mathrm{~nm}$ in hydrodynamic diameter with a surface charge of $49.3 \pm 0.8 \mathrm{mV}$ and polydispersity of $0.19 \pm 0.02$. These particles also consisted of $1.2 \mathrm{pmol}$ of siRNA per $\mu \mathrm{g}$ HA/siRNA layer, but with the incorporation of an additional layer of siRNA, we were able to inject 6417 ng siRNA per mouse eye.

Treatment of the eyes with nanoparticles containing the enhanced siRNA load for 7 days effectively reduced Sparc mRNA levels by mean 2.04-fold $(p=0.0038)$ compared with the scrambled controls (figure 1B). Importantly, decrease in Sparc mRNA expression was associated with significant reduction in Col1a1 transcript expression by mean 2.52fold $(\mathrm{p}=0.00034)$ relative to scramble controls. Fn1 and Mmp14 transcripts were also significantly reduced by 2.89 fold $(p=0.0081)$ and 2.23 -fold $(p=0.029)$, respectively, in the siSparc-treated tissues (figure 1B). We further determined that siSparc treatment also resulted in reduction of Sparc transcript expression on day 14 postsurgery (figure 1C). At this latter time point, Sparc transcript levels remained significantly reduced by 1.39 -fold $(\mathrm{p}=0.049)$. However, there was no corresponding reduction of Col1a1, Fn1 or Mmp14 mRNA expression on day 14 (figure 1C).

At the protein level, both SPARC and COL1A1 were significantly and consistently reduced in tissues treated with siSparc (figure 2). After 7 and 14 days, SPARC protein levels were reduced by mean 1.41 -fold $(\mathrm{p}=0.044)$ (figure $2 \mathrm{~A})$ and 1.89 fold $(p=0.049)$ (figure $2 B)$, respectively. COL1A1 protein was also reduced by mean 2.34 -fold $(p=0.014)$ and 2.41 -fold $(\mathrm{p}=0.017)$ on treatment with siSparc-loaded nanoparticles for 7 and 14 days, respectively. On the other hand, FN1 was only significantly reduced in tissues 7 days after treatment with siSparc, while in the extended period of 14 days, FN1 was no longer suppressed (figure 2A,B). MMP-14 protein levels were not significantly altered by siSparc treatment at both time points. Collectively, these data indicate that siRNA delivered via expanded LbL nanoparticles has the capacity to effectively suppress target (SPARC) and closely associated downstream target (COL1A1) expression for at least 14 days in vivo, while the impact on other indirect targets, including FN1 and MMP14, was less effective.

\section{Imaging the operated conjunctiva treated with siRNA-loaded nanoparticles}

To visualise the tissue response to $\mathrm{LbL}$ nanoparticle-facilitated siRNA treatment, we imaged the live, operated mouse conjunctiva on days $0,2,7$ and 14 after injection with either 


\section{A HA | ARG | siSparc | ARG | siSparc |ARG}
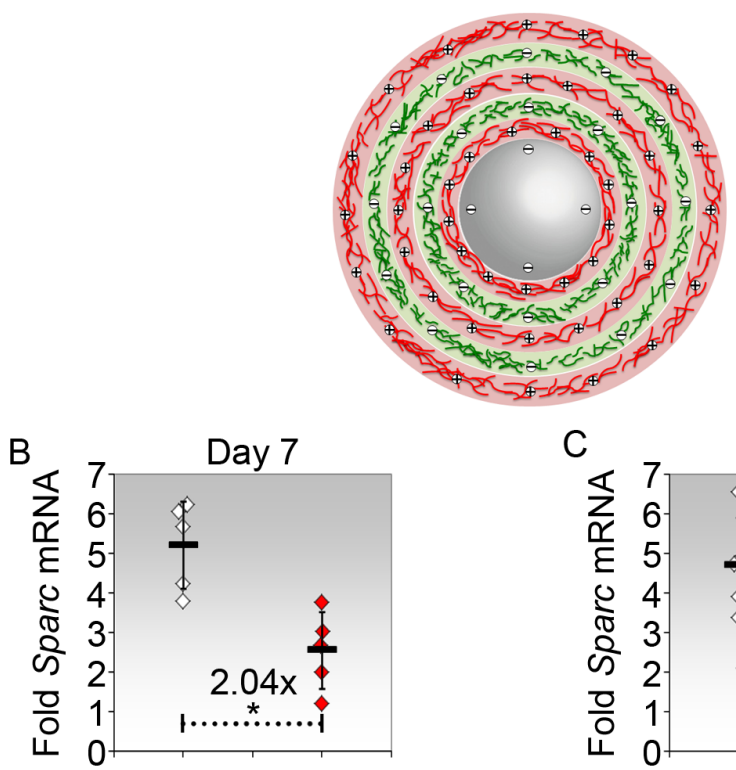

C
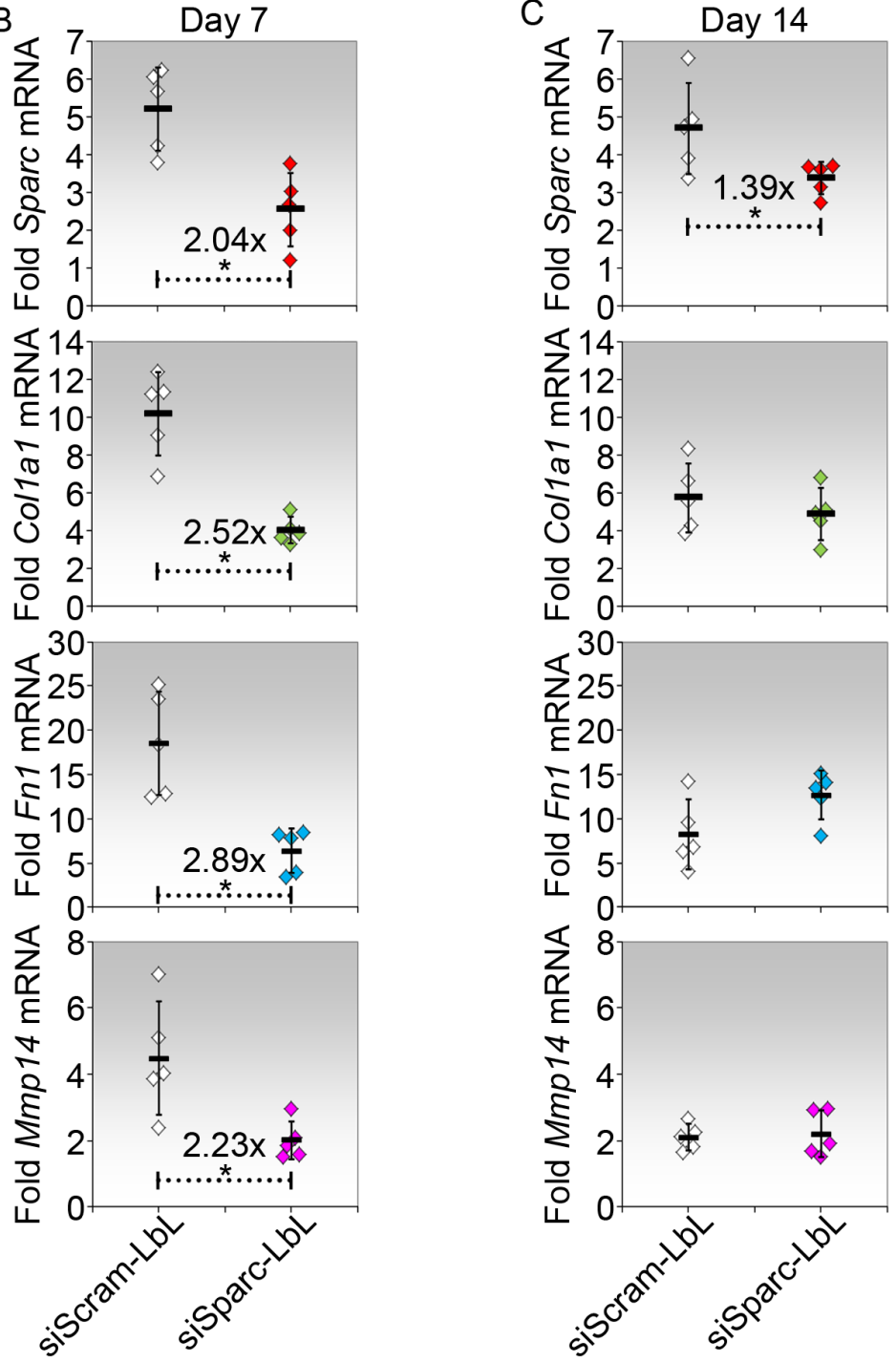

Figure 1 Expression of Sparc and Sparc-regulated transcripts in the mouse model of conjunctival scarring treated with small interfering RNA. (A) Schematic showing the layer composition of five-layer layer-by-layer (LbL) nanoparticles containing two layers of siSparc. (B) Real-time PCR analysis of Sparc, Col1a1, Fn1 and Mmp14 mRNA expression on treatment with the indicated nanoparticles for 7 days. Data shown are the fold changes in mRNA expression in the operated versus paired contralateral unoperated tissues and include the mean fold change \pm SD. Each symbol represents a pooled group of three eyes. Fold change comparing the two treatments calculated from the means of the two groups is also shown. (C) Real-time PCR analysis of Sparc, Col1a1, Fn1 and Mmp14 mRNA expression on treatment with the indicated nanoparticles for 14 days. Data shown were calculated as in (B). ${ }^{*} \mathrm{P}<0.05$ comparing siScram with siSparc treatment. ARG, arginine; HA, hydroxyapatite; +, positive cationic charge; -, negative anionic charge. 
A

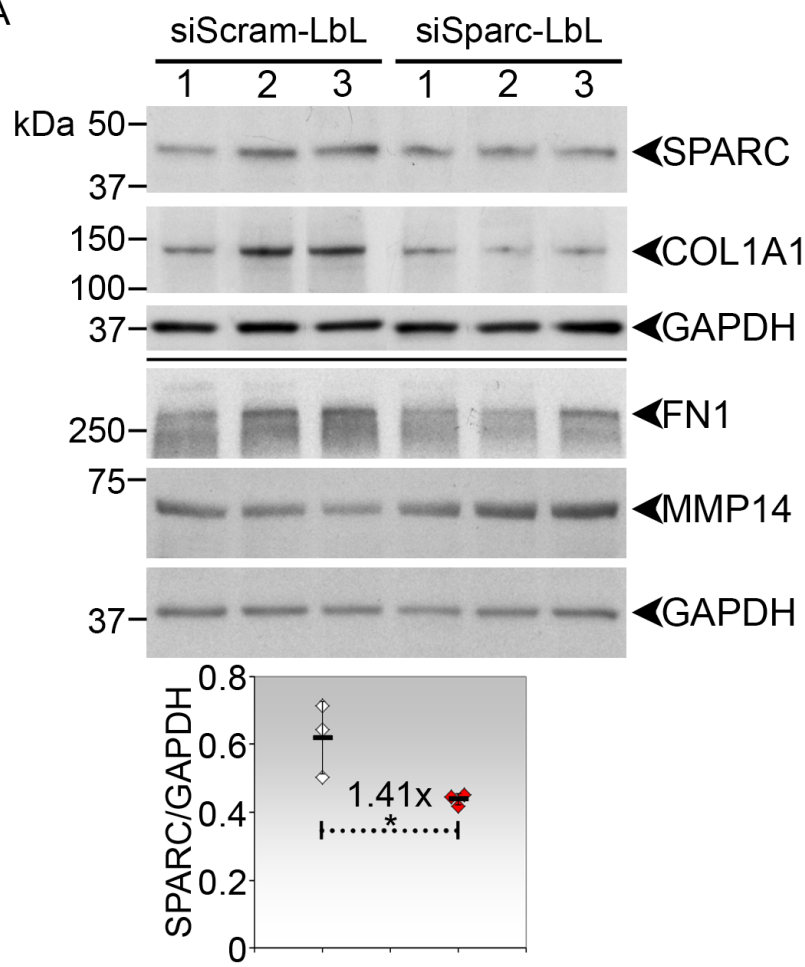

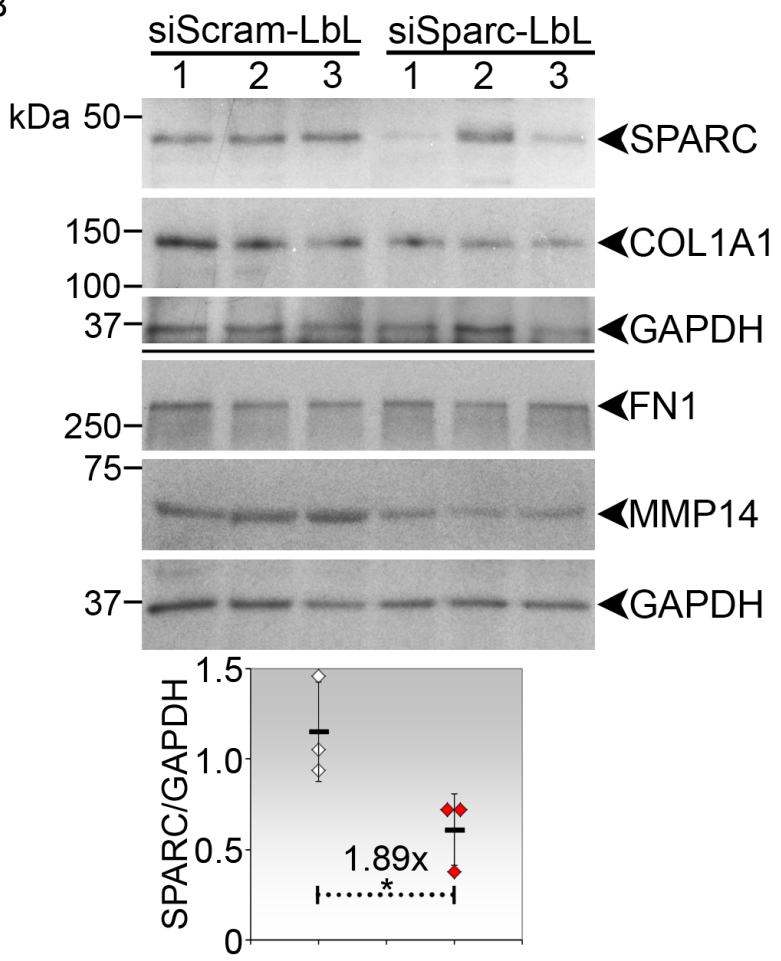
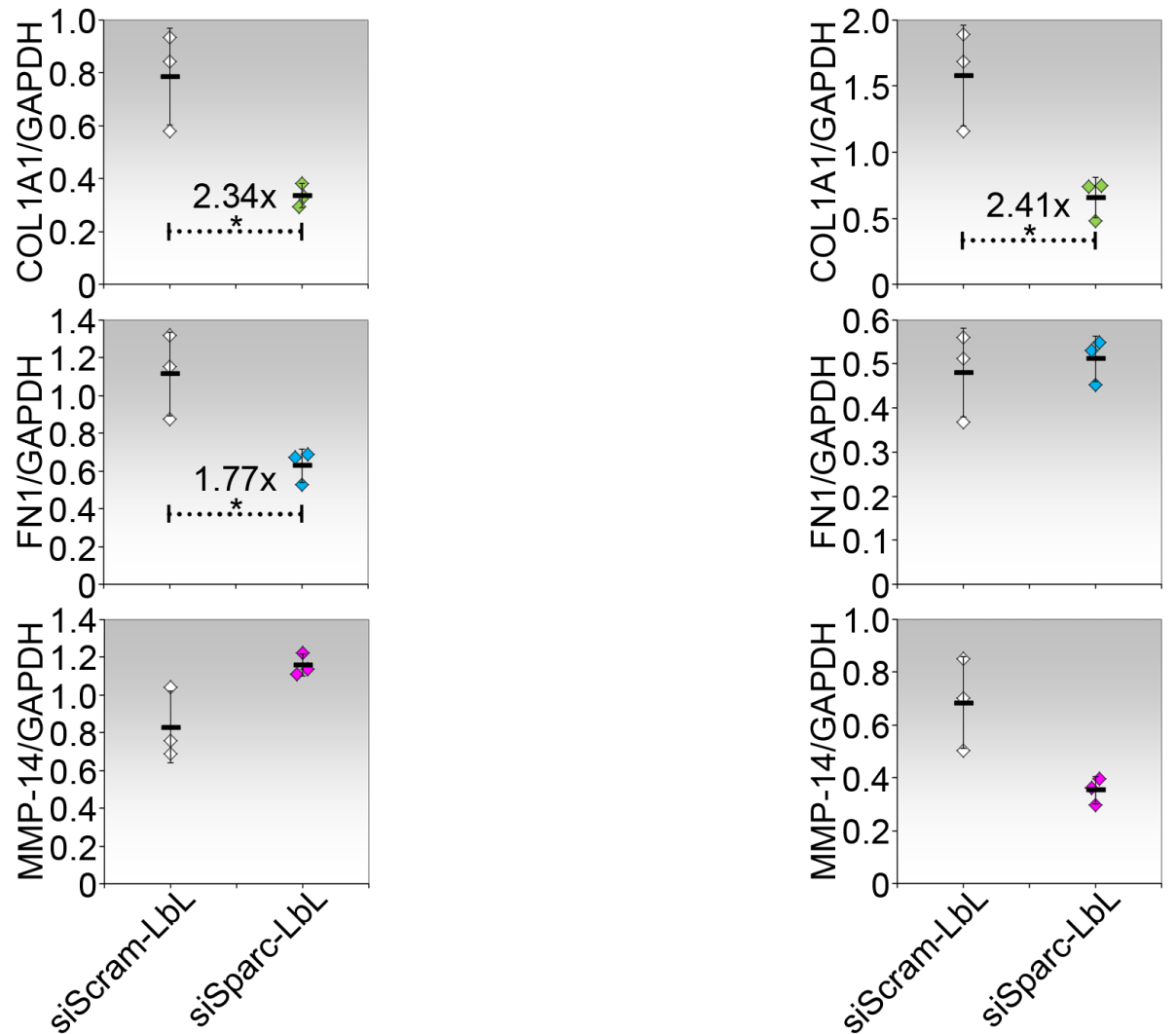

Figure 2 Expression of secreted protein acidic cysteine (SPARC) and SPARC-regulated proteins in the mouse model of conjunctival scarring treated with small interfering RNA. (A) Immunoblot analyses of SPARC, collagen 1A1 (COL1A1), fibronectin 1 (FN1) and matrix metalloproteinase (MMP)14 expression on treatment with either siScram or siSparc for 7 days. (B) Immunoblot analyses of SPARC, COL1A1, FN1 and MMP-14 expression on treatment with either siScram or siSparc for 14 days. Densitometry value of each protein band, expressed as ratio with glyceraldehyde 3-phosphate dehydrogenase (GAPDH) from the same immunoblot, is shown below the immunoblots. The mean fold change \pm SD of the three densitometric ratios for each condition is shown. Fold change comparing the two treatments calculated from the means of the two groups is also shown. ${ }^{*} \mathrm{P}<0.05$ comparing siScram with siSparc treatment. 


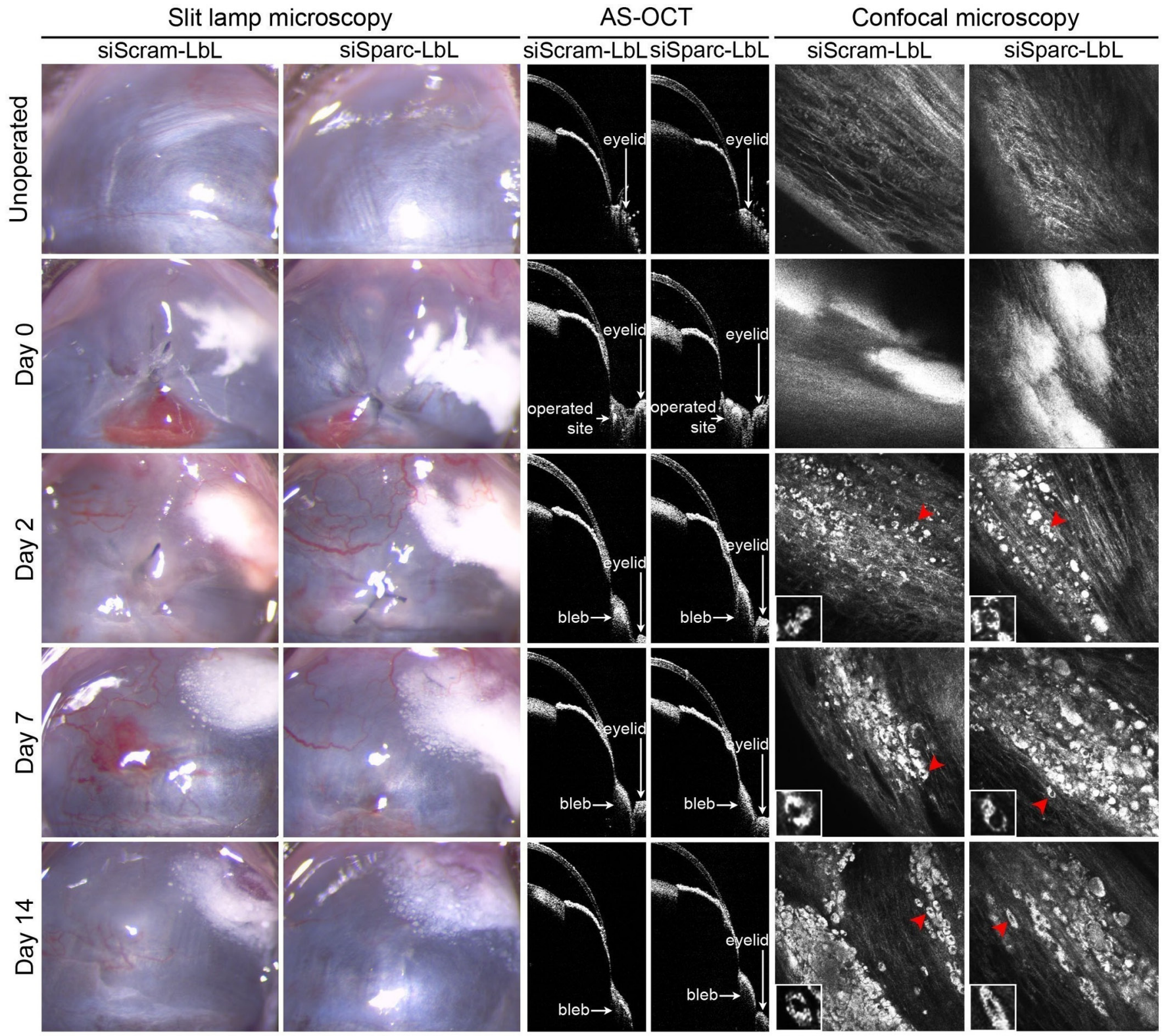

Figure 3 Microscopic analysis of live mouse eyes treated with small interfering RNA-loaded layer-by-layer (LbL) nanoparticles. Experimental surgery was performed on the left eye of each mouse followed by injection with either siScram-loaded or siSparc-loaded nanoparticles. The same operated eye was imaged by slit lamp, anterior segment-optical coherence tomography (AS-OCT) and confocal microscopy on day 0 (immediately following surgery and nanoparticle injection) and days 2, 7 and 14 postsurgery. Insets of confocal images are magnified views of areas indicated by the red arrowheads.

siScram-loaded or siSparc-loaded nanoparticles (figure 3). The injected nanoparticles were conspicuous as a white amorphous substance comprising coalesced nanoparticles when visualised by both slit-lamp microscopy and AS-OCT. Slit-lamp microscopy revealed no overt local conjunctival tissue reaction or increased vascularity beyond what is normally observed as a result of the surgery per se at any of the indicated time points. AS-OCT also did not show any segregation of the surrounding conjunctival tissue from the optically dense nanoparticle material, suggesting that the nanoparticles were well tolerated in the treated tissue. In confocal images, the nanoparticles were observable at first as undefined, large, opaque masses immediately postinjection (day 0 ) and as dense white specks or lumps in the tissue as they scattered at later times. Interestingly, the nanoparticles can be visualised within numerous cell-like structures from day 2 and remained visible in these structures on day 14 postsurgery (red arrowheads and insets, figure 3). These observations suggest that the nanoparticles, loaded with siRNAs, were taken up and retained by cells in the treated tissue for at least 2 weeks, providing support for the capacity of these nanoparticles to successfully deliver siRNA into the cells.

Histochemical evaluation of the day 14 operated conjunctiva treated with siRNA-loaded nanoparticles

We further investigated whether a foreign body reaction may have developed against the siRNA-loaded LbL nanoparticles by examining the treated tissue pathology. In corroboration with the live imaging observations above, overt tissue reaction was not observed in the histological sections of the operated conjunctivas treated with nanoparticles loaded with either scrambled or 


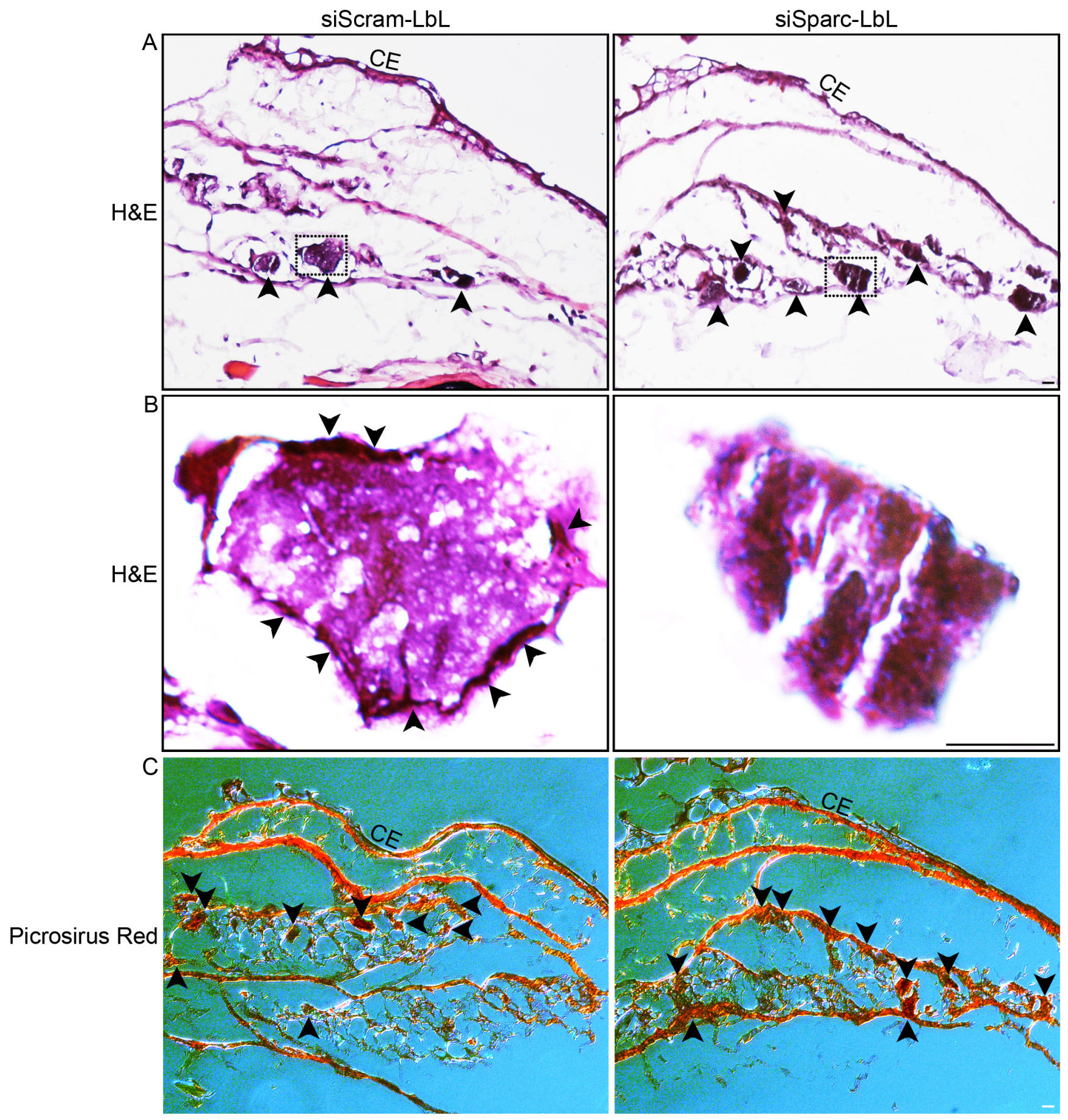

Figure 4 Histochemical analysis of the operated conjunctiva treated with small interfering RNA-loaded layer-by-layer (LbL) nanoparticles for 14 days. (A) H\&E-stained operated conjunctiva treated with siScram-LbL or siSparc-LbL nanoparticles. (B) Higher magnification of the dotted area in the respective $\mathrm{H} \& \mathrm{E}$-stained operated conjunctiva. Arrowheads indicate darker-staining fibre-like structures on the periphery of the coalesced nanoparticles. (C) Picrosirus red-stained operated conjunctiva treated with siScram-LbL or siSparc-LbL nanoparticles. Images, visualised by polarised microscopy, are shown at high exposure to improve visualisation of the collagen matrix. (A and C) Arrowheads indicate the positions of some dyeretaining patches of coalesced nanoparticles. Scale bars $=10 \mu \mathrm{m}$. CE, conjunctival epithelium.

Sparc siRNAs for 14 days (figure 4A). The LbL nanoparticles appeared as relatively small patches of coalesced particles with intense absorption of the histological dyes (figure 4A, arrowheads). At higher magnification, LbL nanoparticles loaded with siScram seemed to be surrounded by darker-staining fibres (figure 4B, arrowheads). In comparison, we did not detect such fibres around a similarly sized patch of aggregated siSparc-loaded LbL nanoparticles (figure 4B). Histochemical staining of the operated and treated conjunctiva with picrosirius red revealed a similar overall tissue appearance (figure 4C). As before, the LbL nanoparticles were conspicuous as they were also saturated with this collagen-staining dye (figure 4C, arrowheads). Notably, 
collagen fibres seemed to penetrate the area containing LbL nanoparticles normally, suggesting that the nanoparticles did not perturb the restoration of the collagen matrix during wound healing. Moreover, the nanoparticles appeared to have settled on collagen fibres which may serve as scaffolds for stable deposition. These observations suggest that treatment with siRNAloaded LbL nanoparticles did not provoke an overt foreign body or tissue response in the operated conjunctiva and allow normal matrix reformation, properties which are beneficial towards in vivo application in targeted therapy.

\section{Immunofluorescence evaluation of the day 14 operated conjunctival extracellular matrix treated with siRNA-loaded nanoparticles}

We also examined the operated conjunctival extracellular matrix by immunofluorescence analyses. As before, the LbL nanoparticles in the cryosections were readily visualised as patches of coalesced particles of varying sizes under brightfield microscopy (figure 5). Immunostaining for SPARC revealed conspicuously fewer positive staining areas in the operated conjunctiva treated with siSparc than that treated with siScram (figure 5A), confirming the reduction of SPARC protein expression with this treatment. Fewer COL1A1-positive staining areas may also be observed with siSparc treatment, although less obvious (figure 5B). At higher magnification, a patch of aggregated siScram-loaded LbL nanoparticles can be visualised to be surrounded by COL1A1 (figure 5B, left, inset), suggesting fibrotic encapsulation of the coalesced nanoparticles. In comparison, a patch of coalesced siSparc-loaded LbL nanoparticles contained less enveloping COL1A1 (figure 5B, right, inset). The distribution of FN1 (figure 5C) and MMP-2 (figure 5D) did not appear to be obviously different between the two treatments. These observations confirmed that siSparc treatment maintained conspicuously less SPARC deposition in the conjunctiva 14 days after experimental surgery. Moreover, siSparc treatment may also be advantageous for controlling collagen encapsulation of the delivery nanoparticles.

\section{Toxicity evaluation of the operated conjunctiva treated with siRNA-loaded nanoparticles}

To evaluate long-term tissue toxicity of Sparc silencing, apoptotic cells in day 14 operated conjunctival tissues were quantitated by flow cytometry (figure 6). Compared with the experimental surgery alone, the injection of nanoparticles loaded with either scrambled or Sparc siRNAs did not significantly increase the number of annexin V-positive cells ( $p>0.05$, figure 6 ) or cause loss of overall cell viability ( $>0.05$, figure 5 ). Moreover, specific treatment with siSparc did not induce significant apoptosis or change the level of cellular viability in the operated conjunctivas compared with treatment with scramble siRNA $(\mathrm{p}>0.05)$. Hence, targeting Sparc expression in the mouse conjunctiva is a safe antifibrotic therapeutic strategy.

\section{DISCUSSION}

This study demonstrates that effective downregulation of a specific gene in the postoperative conjunctiva, in association with its downstream targets, can be achieved via siRNA. Furthermore, our data show that targeted therapy via siRNA loaded on nanoparticles did not cause tissue cell death or overt adverse tissue responses. This proof-of-concept study supports the application of genetic inhibition as effective and safe targeted therapy for treating fibrosis post-GFS.
In this study, we opted to investigate SPARC as the molecule of interest. SPARC has the attributes of a bona fide target molecule for antifibrotic therapeutic development. First of all, SPARC expression features prominently in tissues undergoing turnover such as during development, wound healing and in diseased states, particularly fibrosis. ${ }^{14-20}$ We have also observed increased SPARC expression in the mouse model of conjunctival scarring. ${ }^{7}$ The specific induction of SPARC in activated tissues implies that this molecule may not be critical for normal tissue function in the steady state but is required when tissue turnover occurs. Hence, reducing SPARC expression is not expected to have adverse effects on normal tissues.

Second, SPARC is an ECM-modulatory molecule, being conspicuously involved in the regulation of collagen deposition, both quantitatively and qualitatively in terms of fibril characteristics. ${ }^{11}$ In fact, the ability of SPARC to bind collagen as well as modulate its production and assembly is believed to be key to its functional properties. ${ }^{21}$ It is speculated to behave as a collagen chaperone to ensure that only correctly folded procollagen molecules exit the cell. ${ }^{22}$ As proof of its collagen-regulatory activity, SPARC deficiency is associated with defective ECM architecture and composition. ${ }^{23-26}$ We have also previously reported that SPARC deficiency resulted in altered collagen fibrillogenesis in the eye ${ }^{9}{ }^{10}$ and delayed fibrosis during wound healing. ${ }^{9}$ Thus, SPARC is strongly implicated as a protein involved in mediating fibrosis by regulating collagen production and assembly in the ECM. ${ }^{27}$ This knowledge is critical to the evaluation of Sparc silencing as a successful therapeutic approach. We were able to confirm that SPARC siRNA effectively inhibited SPARC expression (primary target) in the treated conjunctiva, as well as demonstrate the concomitant suppression of collagen as its major downstream, indirect target. The pathology of the siSparc-treated operated conjunctivas was especially revealing in showing reduced accumulation of not only SPARC but also lesser collagen fibres encapsulating the coalesced nanoparticle patches. These data therefore strongly support the specificity, effectiveness and efficiency of this therapeutic system.

Although we have previously demonstrated successful gene silencing using LbL nanoparticles as nanocarriers for siRNA in vitro, ${ }^{13}$ we found in this study that expansion of the LbL format was required to deliver sufficient siRNA in vivo for effective gene silencing. The addition of two more layers did not dramatically alter the hydrodynamic size of the nanoparticles, nor did it impede their accumulation in cell-like structures or caused an overt tissue response in the conjunctiva. The slit-lamp images initially indicated that the nanoparticles were mainly confined in the tissue as a single large mass in the early days post-treatment, but they appeared to progressively scatter with time. Indeed, histological examination revealed aggregated nanoparticles distributed in localised patches of uneven sizes throughout the 14-day operated conjunctiva. This pattern of distribution into smaller parts probably prevented the development of a largescale foreign body reaction as some tissue reaction on a small scale, in the form of collagen fibres enveloping some patches of coalesced nanoparticle, was certainly observed. The suppression of collagen deposition by siSparc is particularly advantageous in this respect since the formation of collagen encapsulation appeared to be repressed, which in turn may enhance the effectiveness of the delivery vehicle. Moreover, the aggregated patches may help preserve the integrity of the siRNA within and so help prolong the lifespan of the gene silencing effects. Hence, the primary reasons driving the in vivo Sparc silencing effectiveness may be attributed to increased quantity of siRNA being delivered to the conjunctiva with the expanded LbL nanoparticle format 


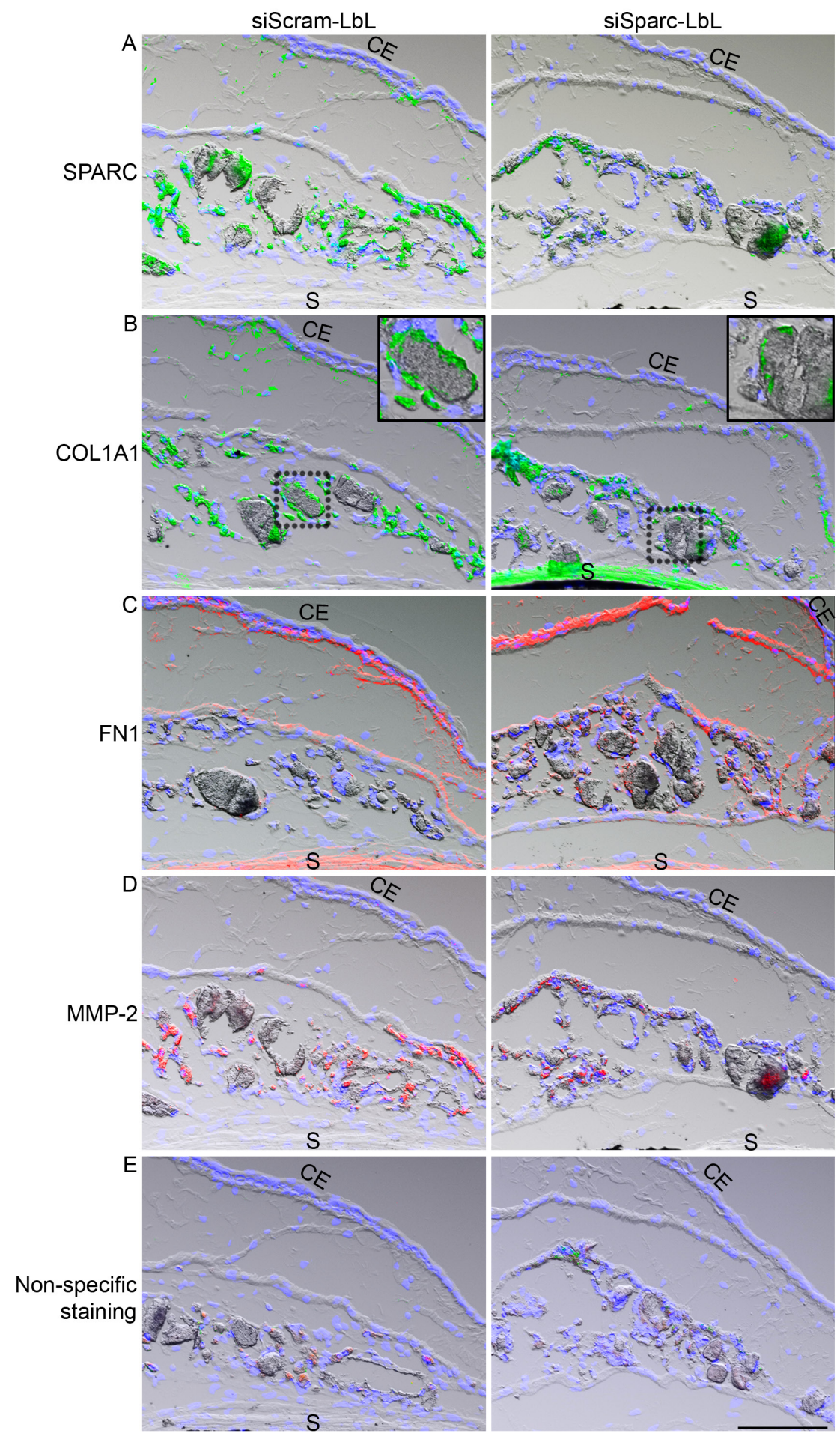

Figure 5 Immunofluorescence analysis of the operated conjunctiva treated with small interfering RNA-loaded layer-by-layer (LbL) nanoparticles for 14 days. (A) Operated conjunctivas treated with siScram-LbL or siSparc-LbL nanoparticles and immunostained with secreted protein acidic cysteine (SPARC) antibody. SPARC-positive areas were visualised as green fluorescence. (B) Operated conjunctivas treated with siScram-LbL or siSparc-LbL nanoparticles and immunostained with collagen $1 \mathrm{~A} 1$ (COL1A1) antibody. COL1A1-positive areas were visualised as green fluorescence. Higher magnification of the dotted box area is shown in the inset. (C) Operated conjunctivas treated with siScram-LbL or siSparc-LbL nanoparticles and immunostained with fibronectin 1 (FN1) antibody. FN1-positive areas were visualised as red fluorescence. (D) Operated conjunctivas treated with siScram-LbL or siSparc-LbL nanoparticles and immunostained with matrix metalloproteinase (MMP)-2 antibody. MMP-2-positive areas were visualised as red fluorescence. (E) Operated conjunctivas treated with siScram-LbL or siSparc-LbL nanoparticles and immunostained with secondary antibodies only. Non-specific staining was visualised as both red and green fluorescence. Nuclei were visualised by 4',6-diamidino-2-phenylindole staining (blue). All images were taken at the same exposure and microscopy settings. The fluorescent image of each sample, photographed with the appropriate filter, was overlaid with the image taken in brightfield to allow visualisation of the nanoparticles. Scale bar $=100 \mu \mathrm{m}$. CE, conjunctival epithelium; S, sclera. 

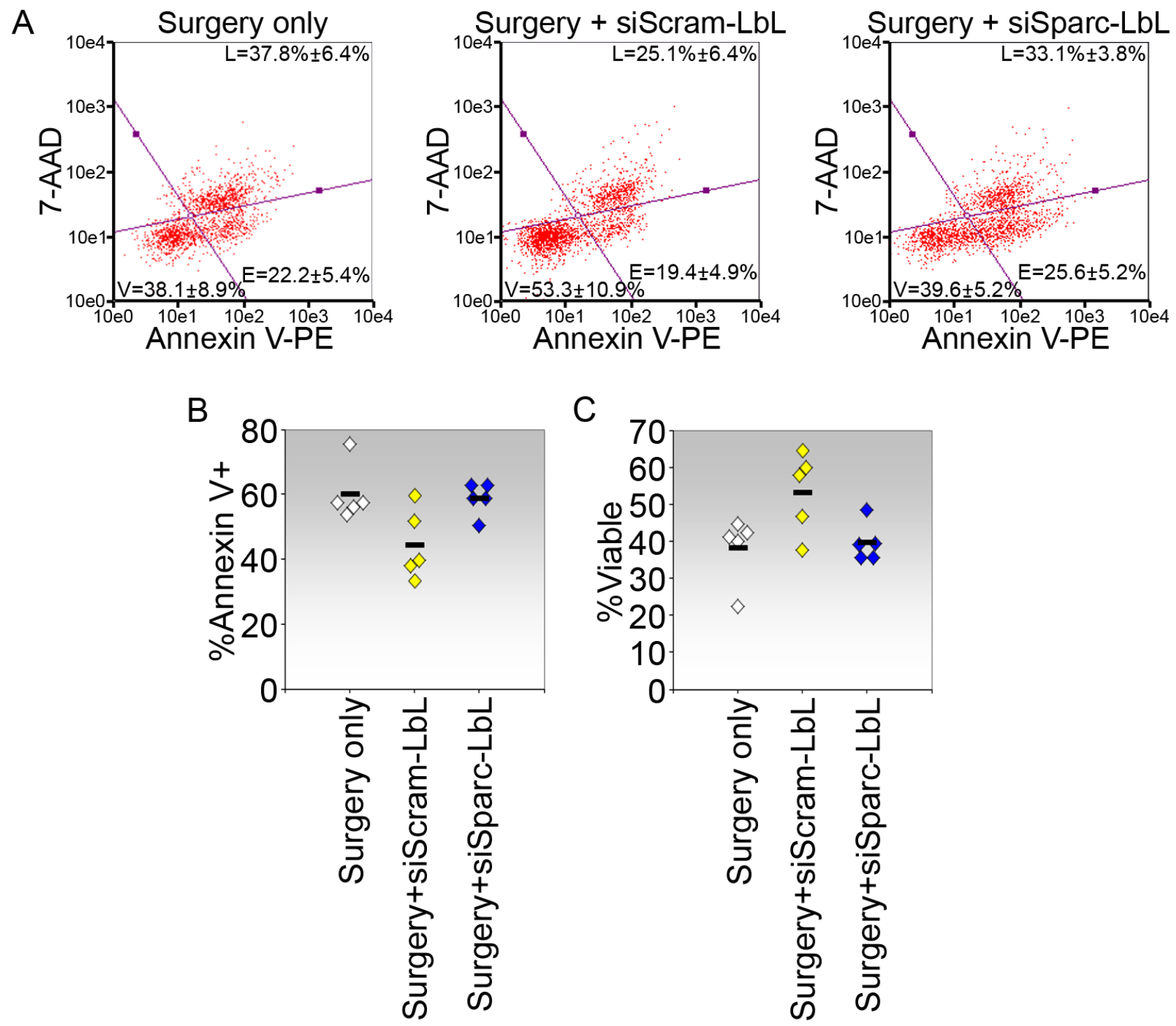

Figure 6 Tissue toxicity analysis of the operated conjunctiva treated with small interfering RNA-loaded layer-by-layer (LbL) nanoparticles for 14 days. (A) Representative scatter plots of annexin V-stained samples from experimental surgery alone (left), surgery cum treatment with siScram-loaded LbL nanoparticles (middle) and surgery cum siSparc-loaded LbL nanoparticles (right). Indicated values denote the percentage mean \pm SD of cells in the associated quadrant $(n=5)$. (B) Percentage of annexin V-positive cells in operated conjunctival tissues treated as indicated. (C) Percentage of viable cells in operated conjunctival tissues treated as indicated. Each symbol represents a pool of tissues from three eyes of independent animals.E, early apoptotic cells; L, late apoptic or dead cells; V, viable cells.

and to subdued tissue reaction and/or protection of siRNA facilitated by the distribution of the nanoparticles in small coalesced patches.

The capacity of the nanoparticle system to achieve sustained gene downregulation in the conjunctiva has a clear advantage in that multiple injections of these siRNA-loaded drugs are potentially not required to maintain the therapeutic effect for an extended period of time postsurgery. However, there was an apparent reduction in efficiency of gene silencing with time, which we presume to be related to depletion and/or loss of siRNA stability in the tissue. The lower efficiency of Sparc silencing at the later time point was associated with loss of significant reduction in Col1a1 transcription, implying that a minimum threshold delivery of siRNA or Sparc silencing was required to generate a significant downstream effect. The same may be said for regulation of the other extracellular matrix genes, Fn1 and Mmp14, which were also suppressed by SPARC inhibition at the mRNA level in the short term, but their regulation at the protein level, and in the extended period post-treatment, appeared to be less effective. Hence, the capacity to deliver large quantities of siRNA, and for a prolonged period, is clearly a factor that needs to be considered in future designs of siRNA delivery systems. Nevertheless, our data indicate that active target gene silencing is achievable for at least 2 weeks post-treatment.

Curiously, the similar levels of downregulation of both SPARC and COL1A1 proteins in the treated tissues on both 7 and 14 
days did not correlate with alterations in their transcript levels caused by siSparc treatment. This suggests that reduction of these two proteins, attained at earlier times, was maintained till at least day 14, irrespective of their mRNA levels. We speculate that this phenomenon may be influenced by alterations in specific gene activity as wound healing progresses. It is possible that synthesis of these two proteins reduced dramatically from day 7 to day 14 , as suggested by our previous observation that the induction of Col1a1 transcription was highest on day 7 postsurgery but dropped thereafter. ${ }^{28}$ Low production of COL1A1, and likely SPARC, coupled with potentially low degradation, may then lead to minimal additional accumulation of these two proteins at the later time point so that the levels measured on day 14 were mainly derived from synthesis at earlier times. This phenomenon, although dependent on the biological properties of the specific genes/proteins concerned, is highly beneficial in terms of antifibrotic therapy and does suggest that at least for SPARC and COL1A1, long-term inhibition at the transcript levels may not be necessary to achieve sustained therapeutic reduction of collagen accumulation in the remodelling tissue.

In summary, we show in this proof-of-principle study that targeted therapy via siRNA is a promising application in improving GFS success along with a reduced surgical morbidity. By targeting SPARC as well as a range of potential ECM targets associated with fibrosis, the data presented here advances oligonucleotide therapeutics as potentially effective antifibrotic therapies for postoperative conjunctival fibrosis. Moreover, we predict that advanced, compact nanocarriers with the capacity to deliver large loads of siRNA in a single dose will be extremely useful for the development of genetic inhibition as targeted therapy in the eye.

Acknowledgements We would like to thank Alice $\mathrm{Ng}$ and Nyein Chan Lwin from Singapore Eye Research Institute for assistance with animal experiments.

Contributors LFS analysed the data and wrote the manuscript. LFS, YFT, LZT and SCWL performed the experiments. SV and TTW conceptualised the study. TTW edited and approved the manuscript.

Funding This work was funded by a Clinician Scientist Award grant (NMRC/ CSA-SI/0001/2015) and by the Singapore National Research Foundation under its Translational and Clinical Research (TCR) Programme (NMRC/TCR/002-SERI/2008; NMRC/TCR/008-SERI/2013) to TTW, both administered by the Singapore Ministry of Health's National Medical Research Council. Animal studies were funded by a SingHealth Foundation Research Grant (SHF/FG583P/2014) to LFS and partially funded by the SERI core grant (NMRC/CG/015/2013).

Competing interests None declared.

Patient consent Not required.

Ethics approval Institutional Animal Care and Use Committee (IACUC).

Provenance and peer review Not commissioned; externally peer reviewed.

Open access This is an Open access article distributed in accordance with the Creative Commons Attribution Non Commercial (CC BY-NC 4.0) license, which permits others to distribute, remix, adapt, build upon this work non-commercially, and license their derivative works on different terms, provided the original work is properly cited, appropriate credit is given, any changes made indicated, and the use is non-commercial. See: http://creativecommons.org/licenses/by-nc/4.0/.

\section{REFERENCES}

1 Morgan WH, Yu DY. Surgical management of glaucoma: a review. Clin Exp Ophthalmol 2012:40:388-99.
2 Radcliffe NM. Trabeculectomy revision as a treatment for failed trabeculectomy. Glaucoma Today 2010(Fall):25-8.

3 Yamamoto T, Sawada A, Mayama C, et al. The 5-year incidence of bleb-related infection and its risk factors after filtering surgeries with adjunctive mitomycin $\mathrm{C}$ : collaborative bleb-related infection incidence and treatment study 2. Ophthalmology 2014;121:1001-6.

4 Hueber A, Esser JM, Kociok N, et al. Mitomycin C induces multidrug resistance in glaucoma surgery. Graefes Arch Clin Exp Ophthalmol 2008;246:297-304.

5 Brekken RA, Sage EH. SPARC, a matricellular protein: at the crossroads of cell-matrix. Matrix Biol 2000;19:569-80.

6 Bornstein P, Sage EH. Matricellular proteins: extracellular modulators of cell function. Curr Opin Cell Biol 2002;14:608-16.

7 Seet LF, Finger SN, Chu SW, et al. Novel insight into the inflammatory and cellular responses following experimental glaucoma surgery: a roadmap for inhibiting fibrosis. Curr Mol Med 2013;13:911-28.

8 Basu A, Kligman LH, Samulewicz SJ, et al. Impaired wound healing in mice deficient in a matricellular protein SPARC (osteonectin, BM-40). BMC Cell Biol 2001;2:15.

9 Seet LF, Su R, Barathi VA, et al. SPARC deficiency results in improved surgical survival in a novel mouse model of glaucoma filtration surgery. PLOS One 2010;5:e9415.

$10 \mathrm{Ho} \mathrm{H}, \mathrm{Htoon} \mathrm{HM}$, Yam GH, et al. Altered anterior segment biometric parameters in mice deficient in SPARC. Invest Ophthalmol Vis Sci 2017:58:386-93.

11 Bradshaw AD. The role of SPARC in extracellular matrix assembly. I Cell Commun Signal 2009;3:239-46.

12 Seet LF, Su R, Toh LZ, et al. In vitro analyses of the anti-fibrotic effect of SPARC silencing in human Tenon's fibroblasts: comparisons with mitomycin C. J Cell Mol Med 2012;16:1245-59.

13 Tan YF, Mundargi RC, Chen MH, et al. Layer-by-layer nanoparticles as an efficient siRNA delivery vehicle for SPARC silencing. Small 2014;10:1790-8.

14 Zhou X, Tan FK, Reveille JD, et al. Association of novel polymorphisms with the expression of SPARC in normal fibroblasts and with susceptibility to scleroderma. Arthritis Rheum 2002;46:2990-9.

15 Zhou X, Tan FK, Guo X, et al. Attenuation of collagen production with small interfering RNA of SPARC in cultured fibroblasts from the skin of patients with scleroderma. Arthritis Rheum 2006;54:2626-31.

16 Kuhn C, Mason RJ. Immunolocalization of SPARC, tenascin, and thrombospondin in pulmonary fibrosis. Am J Pathol 1995;147:1759-69.

17 Pichler RH, Hugo C, Shankland SJ, et al. SPARC is expressed in renal interstitial fibrosis and in renal vascular injury. Kidney Int 1996;50:1978-89.

18 Kanauchi M, Nishioka M, Dohi K. Secreted protein acidic and rich in cysteine (SPARC) in patients with diabetic nephropathy and tubulointerstitial injury. Diabetologia 2000:43:1076-7

19 Frizell E, Liu SL, Abraham A, et al. Expression of SPARC in normal and fibrotic livers. Hepatology 1995;21:847-54.

20 Dhore CR, Cleutjens JP, Lutgens E, et al. Differential expression of bone matrix regulatory proteins in human atherosclerotic plaques. Arterioscler Thromb Vasc Biol 2001;21:1998-2003.

21 Rentz TJ, Poobalarahi F, Bornstein P, et al. SPARC regulates processing of procollagen I and collagen fibrillogenesis in dermal fibroblasts. J Biol Chem 2007;282:22062-71

22 Martinek N, Shahab J, Sodek J, et al. Is SPARC an evolutionarily conserved collagen chaperone? J Dent Res 2007;86:296-305.

23 Bradshaw AD, Puolakkainen P, Dasgupta J, Wight TN, et al. SPARC-null mice display abnormalities in the dermis characterized by decreased collagen fibril diameter and reduced tensile strength. J Invest Dermatol 2003;120:949-55.

24 Delany AM, Amling M, Priemel M, et al. Osteopenia and decreased bone formation in osteonectin-deficient mice. J Clin Invest 2000;105:915-23.

25 Bradshaw AD, Puolakkainen P, Dasgupta J, et al. SPARC-null mice display abnormalities in the dermis characterized by decreased collagen fibril diameter and reduced tensile strength. J Invest Dermatol 2003;120:949-55.

26 Strandjord TP, Madtes DK, Weiss DJ, et al. Collagen accumulation is decreased in SPARC-null mice with bleomycin-induced pulmonary fibrosis. Am J Physiol 1999;277:L628-L635.

27 Trombetta-Esilva J, Bradshaw AD. The function of SPARC as a mediator of fibrosis. Open Rheumatol I 2012;6:146-55.

28 Seet LF, Toh LZ, Chu SWL, et al. Upregulation of distinct collagen transcripts in postsurgery scar tissue: a study of conjunctival fibrosis. Dis Model Mech 2017;10:751-60. 